\title{
Der Zusammenhang von Lerngelegenheiten und Fachwissen im Lehramtsstudium - eine Frage der Intensität?
}

\section{Johannes Osterberg, Marc Motyka, Johannes Gerken und Frank Lipowsky}

Dieser Beitrag befasst sich mit dem Zusammenhang zwischen den Lerngelegenbeiten im bildungswissenschaftlichen Teil des Lehramtsstudiums und dem pädagogisch-psychologischen Professionswissen von Lehramtsstudierenden. Anhand einer Stichprobe von $N=109$ Kasseler Lehramtsstudierenden kann gezeigt werden, dass die Lerngelegenheiten der Studierenden im bildungswissenschaftlichen Studium erfasst als behandelte Inhalte - das pädagogisch-psychologische Wissen voraussagen und dass die Vorhersagekraft zunimmt, wenn die Lerngelegenheiten einen engeren inhaltlichen Zusammenhang mit dem Wissen aufweisen. Zudem kann anknüpfend an Angebots-Nutzungs-Modelle der Professionalisierungsforschung nachgewiesen werden, dass die Intensität der Auseinandersetzung mit den Lerngelegenheiten den Zusammenhang zwischen Lerngelegenheiten und Wissen mediiert. Die simultane Berücksichtigung von Lerngelegenheiten und Beschäftigungsintensität in diesem Mediationsmodell kann zudem mehr Varianz im pädagogisch-psychologischen Wissen aufklären als die alleinige Berücksichtigung der Lerngelegenheiten.

\section{Einleitung}

Die Bereitstellung von inhaltlich relevanten Lerngelegenheiten, sogenannten opportunities to learn (OTL), gilt als wichtiger Einflussfaktor für das Lernen in Schule, Hochschule und Weiterbildung. Auch in der Lehrerinnen- und Lehrerbildung wächst die Bedeutung, die der Erfassung von Lerngelegenheiten und ihrer Nutzung durch angehende oder bereits im Beruf befindliche Lehrpersonen zugeschrieben wird (Kunter, Kleickmann, Klusmann \& Richter, 2011). Dahinter steht die Überzeugung, dass der Erwerb professionellen Wissens von Lehrpersonen inhaltlich relevante Lerngelegenheiten in Studium und Weiterbildung voraussetzt. Klagen über «schlechte» Lehrpersonen oder einen fehlenden Transfer wissenschaftlicher Erkenntnisse in die Klassenzimmer sind aus dieser Sicht immer auch in Beziehung zu setzen zur Frage, inwieweit im Rahmen der Ausbildung von Lehrpersonen überhaupt entsprechende Lerngelegenheiten vorhanden waren. 
Die bisherige Forschung im Bereich der Lehramtsausbildung kommt allerdings, was den Zusammenhang zwischen bestehenden Lerngelegenheiten im Studium und dem Wissen der Studierenden anbelangt, zu keinen einheitlichen Ergebnissen. Eine Rolle hierbei spielt möglicherweise auch die uneinheitliche Erfassung von Lerngelegenheiten. In diesem Beitrag wird ein Ansatz vorgestellt, bei dem zwischen den von Studierenden behandelten Lerngelegenheiten und der Beschäftigungsintensität im bildungswissenschaftlichen Studium unterschieden wird. Mittels Regressionsanalysen wird in einem ersten Schritt geprüft, inwieweit das Vorkommen von Lerngelegenheiten das pädagogisch-psychologische Wissen vorhersagen kann. Im zweiten Schritt wird untersucht, ob die Beschäftigungsintensität diesen Zusammenhang zwischen Lerngelegenheiten und pädagogisch-psychologischem Wissen vermittelt.

\section{Theoretischer und konzeptioneller Hintergrund}

Die empirische Bildungsforschung beschäftigt sich seit geraumer Zeit mit Determinanten des Lernens. Eine bedeutsame Rolle spielt hierbei die Aufmerksamkeit, die Lernende für die Auseinandersetzung mit Lerninhalten aufbringen. In älteren Modellen der Unterrichtsforschung wird in diesem Zusammenhang von time on task (Carroll, 1963; Harnischfeger \& Wiley, 1977) oder von aktiv genutzter Lernzeit gesprochen. Im Mittelpunkt entsprechender Studien standen vor allem die Fragen, wie aufmerksam die zur Verfügung gestellte Lernzeit von Lernenden genutzt wird und in welchem Ausmass sie den Lernerfolg der Lernenden erklären kann (z. B. Anderson, 1984; Helmke \& Renkl, 1992; Samuels \& Turnure, 1974; Treiber 1982).

Mit der Hinwendung zu kognitiv-konstruktivistischen Theorien hat sich der Forschungsfokus von einer eher verhaltensbezogenen Erfassung der Aufmerksamkeit der Lernenden zu einer stärker inhaltsbezogenen Konzeptualisierung der Auseinandersetzung verschoben, bei der zwischen dem Angebot an inhaltlichen Lerngelegenheiten und seiner Nutzung unterschieden wird. Seinen konzeptionellen Niederschlag findet dies in sogenannten Angebots-Nutzungs-Modellen, die seit längerem in der Schul- und Unterrichtsforschung (z. B. Fend, 2002; Helmke, 2012; Reusser \& Pauli, 2010) und mittlerweile auch in der Forschung zur Lehrerinnen- und Lehrerbildung Anwendung finden (Hascher \& Kittinger, 2014; König, Rothland \& Schaper, 2018; Kunter et al. 2011; Lipowsky, 2014; Mayr, 2012). Mit der Unterscheidung zwischen Angebot und Nutzung wird berücksichtigt, dass zur Verfügung gestellte inhaltliche Lernangebote von den Lernenden sehr unterschiedlich wahrgenommen und genutzt werden können. Für das Lernen spielt deshalb nicht nur das Angebot an Lerngelegenheiten, sondern auch seine Nutzung durch die Lernenden eine entscheidende Rolle. Durch die Befragung von Lernenden lassen sich angebots- und nutzungsseitige Aspekte von Lerngelegenheiten für die empirische Forschung erfassen. 
Bislang konnte sich in der Forschung allerdings noch keine einheitliche Konzeptualisierung von Lerngelegenheiten bzw. OTL durchsetzen. Ursprünglich wurde das OTL-Konzept im Kontext der ersten internationalen Schulleistungsstudien eingeführt. Mit ihnen wurden die in den verschiedenen Ländern behandelten Unterrichtsinhalte identifiziert, um zu ermitteln, welche Inhalte unter Berücksichtigung der Testfairness in den international vergleichenden Leistungstests sinnvollerweise abgefragt bzw. geprüft werden können (McDonnell, 1995). So stellte Husen (1967, S. 162f.) bereits im Kontext der ersten IEA-Studien heraus: "One of the factors which may influence scores on an achievement examination is whether or not the students have had an opportunity to study a particular topic or learn how to solve a particular type of problem ...». In einer aktuellen Konzeptualisierung von OTL wird jedoch nicht nur der inhaltliche Aspekt aufgegriffen, sondern es werden mit der Zeit und der Qualität zwei weitere Dimensionen berücksichtigt. Kurz et al. (2014) definieren OTL als «degree to which a teacher dedicates instructional time and content coverage to the intended curriculum objectives emphasizing high-order cognitive processes, evidence-based instructional practices, and alternative grouping formats» (S. 27).

Zusätzlich zu den zeitlichen, inhaltlichen und qualitativen Aspekten können in Anlehnung an Tynjälä (2008) auch die Kontexte, in die Lerngelegenheiten eingebunden sind, unterschieden werden. Formale Lerngelegenheiten finden in Institutionen statt, die spezifisch gestaltete Lehr-Lern-Settings bereitstellen und hierüber zu formalen Abschlüssen führen. Als nonformale Lerngelegenheiten werden Lernsituationen beschrieben, welche sich ausserhalb eines gesteuerten institutionellen Lehr-Lern-Settings ergeben. Dabei weisen nonformale Lerngelegenheiten, wie etwa Arbeitsgemeinschaften oder Lerngruppen, meist eine enge Verbindung zu den formalen Lerngelegenheiten auf. Die dritte Art der Kontextualisierung stellen informelle Lerngelegenheiten dar, bei denen Lernprozesse stattfinden, die weder durch formale Vorgaben gesteuert sind, noch zwangsläufig zu formalen Bildungsabschlüssen führen. Informelle Lerngelegenheiten grenzen sich von formalen und nonformalen durch ihre meist situative Spontaneität ab und werden deshalb nicht zwangsläufig als Lernprozesse wahrgenommen.

Im Kontext formaler OTL lassen sich mit Kuger (2016) zudem verschiedene Ebenen unterscheiden. Auf struktureller Ebene ergeben sich OTL demnach durch die Anzahl der Stunden oder Credits, die in der Stundentafel für ein Fach oder einen Studienschwerpunkt ausgewiesen sind, und durch das konkrete Curriculum, das sich in Bildungsplänen und Prüfungsvorschriften widerspiegelt. Auf der Mikroebene sind es die von der einzelnen Lehrperson getroffenen didaktischen Entscheidungen bei der Vorbereitung und Durchführung des (Hochschul-) Unterrichts, die die zur Verfügung gestellten Lerngelegenheiten bestimmen. In Hochschulkontexten wird häufig zusätzlich zwischen dem intendierten oder vorgegebenen Curriculum, dem implementierten oder realisierten Curriculum und dem erreichten Curriculum in Form von Lernergebnissen unterschieden (Blömeke, Kaiser \& Lehmann, 2010; McDonnell, 1995; Stancel-Piatak et al., 2013). 


\section{Universitäre Lerngelegenheiten im Lehramts- studium: Relevanz und Forschungsbefunde}

Lerngelegenheiten und ihre Nutzung bilden den Kern sogenannter Angebots-Nutzungs-Modelle, auf die auch in der Professionalisierungsforschung zunehmend rekurriert wird: Im Modell der Determinanten und Konsequenzen der professionellen Kompetenz von Lehrkräften betrachten Kunter et al. (2011) Lerngelegenheiten als eine zentrale Variable für die Professionalisierung von Lehrpersonen. So hängt der Aufbau professioneller Kompetenz in der Modellvorstellung unter anderem von den angebotenen und den durch die (angehenden) Lehrpersonen genutzten Lerngelegenheiten ab. Die angebotenen Lerngelegenheiten bspw. in Form von universitären Lehrveranstaltungen oder in Form von Fort- und Weiterbildungen stellen aus theoretisch-konzeptioneller Sicht eine wichtige Determinante für den Erwerb von Wissen und den Aufbau von Handlungskompetenzen (angehender) Lehrpersonen dar. Dies spiegelt sich u.a. auch im Rahmenmodell der TEDS-M-Studie von Blömeke et al. (2009) und im Untersuchungsdesign der LEK-Studie von König und Seifert (2012) wider.

Welche Befunde liegen zur Vorhersagerelevanz von genutzten Lerngelegenheiten vor? Schmidt, Cogan und Houang (2011) untersuchen auf der Grundlage von TEDS-M-Daten, inwiefern angehende Mathematiklehrkräfte während ihrer Hochschulausbildung unterschiedlich viele Lerngelegenheiten in den Bereichen Fachwissenschaft, Bildungswissenschaften und Fachdidaktik (erfasst als behandelte Themen bzw. Kurse) erhalten haben und inwiefern diese Unterschiedlichkeit mit der Ausprägung der entsprechenden Kompetenzfacetten zusammenhängt. Für die Subgruppe der US-amerikanischen Studierenden konnten mehrebenenanalytisch signifikante Effekte von Lerngelegenheiten auf das mathematische Fachwissen sowie auf das fachdidaktische Wissen nachgewiesen werden.

Ebenfalls auf Grundlage von querschnittlichen Daten der TEDS-M-Studie untersuchten Blömeke, Suhl, Kaiser und Döhrmann (2012) den Zusammenhang von Lerngelegenheiten - erfasst über die Anzahl studierter Lerninhalte - und dem Fachwissen sowie dem fachdidaktischen Wissen von angehenden Mathematiklehrkräften für den Grundschulbereich. Erwartungsgemäss stellten sich die Lerngelegenheiten in der Fachwissenschaft als bedeutsam für den Erwerb mathematischen Fachwissens heraus. Darüber hinaus offenbarten sich fachwissenschaftliche Lerngelegenheiten auch als Prädiktoren für den Erwerb fachdidaktischen Wissens und scheinen demnach die Wirksamkeit fachdidaktischer Lerngelegenheiten zu unterstützen. Die Autorengruppe bilanziert folglich, dass "OTL in mathematics were of outstanding relevance for the outcomes of primary teacher education» (ebd., S. 53).

Im Rahmen der TEDS-LT-Studie von Stancel-Piatak et al. (2013) wurde u.a. das Fachwissen von Lehramtsstudierenden der Fächer Deutsch, Englisch, Mathematik und Pädagogik an acht Hochschulen in Deutschland untersucht. 
In den Fächern Deutsch, Englisch und Mathematik wurden das fachwissenschaftliche und das fachdidaktische Wissen als abhängige Variablen analysiert, in Pädagogik das pädagogische Wissen. Diese Wissensbestände wurden mehrebenenanalytisch durch Lerngelegenheiten vorhergesagt. Die Lerngelegenheiten wurden für jedes Fach als zeitlicher Studieraufwand für vorgeschriebene Lehrveranstaltungen, für Arbeitsgruppen, für freiwillige Lehrveranstaltungen, für Vorund Nachbereitung von Lehrveranstaltungen und für sonstigen studienbezogenen Aufwand erfasst. Insgesamt zeigten sich inkonsistente Ergebnisse zur Vorhersagekraft der über die Dimension Zeit erfassten Lerngelegenheiten. So erwiesen sich die in vorgeschriebenen Lehrveranstaltungen und Arbeitsgruppen investierten Zeitressourcen in den Fächern Englisch und Deutsch teilweise sogar als negative, in Mathematik dagegen als positive Prädiktoren. In Englisch wirkte sich im Gegensatz zu den anderen Fächern die Teilnahme an freiwilligen Lehrveranstaltungen positiv auf das fachdidaktische Wissen aus (Stancel-Piatak et al., 2013). Ein erwartungsgemäss positiver Zusammenhang liess sich zwischen der aufgebrachten Zeit für die Vor- und Nachbereitung von Lehrveranstaltungen und den fachwissenschaftlichen Leistungen in Mathematik feststellen. In einer etwas schwächeren Ausprägung waren auch die Lerngelegenheiten im Fach Pädagogik, gemessen über die Zeit für die Vor- und Nachbereitung der Lehrveranstaltungen, für das pädagogische Wissen prädiktiv, während die anderen vier Zeitvariablen keine Vorhersagekraft für das pädagogische Wissen hatten.

Auch in der LEK-Studie von König, Tachtsoglou und Seifert (2012) wurde der Zusammenhang zwischen erziehungswissenschaftlichen Lerngelegenheiten und dem pädagogischen Professionswissen untersucht. Hierbei wurden mehrere deutsche Hochschulstandorte einbezogen. Das pädagogische Professionswissen wurde über das pädagogische Unterrichtswissen und das bildungswissenschaftliche Wissen und dazugehörende Subfacetten erfasst. Insgesamt konnten König et al. (2012) an mehreren Standorten positive Zusammenhänge zwischen dem Besuch von spezifischen Lehrveranstaltungen und Modulen einerseits und verschiedenen Wissensdimensionen andererseits nachweisen, wenngleich diese Zusammenhänge in vielen Fällen nicht signifikant werden. Die nachgewiesenen signifikanten Zusammenhänge sind jedoch sämtlich positiv. Insbesondere für die Paderborner Stichprobe ergaben sich signifikante Zusammenhänge, was die Autoren mit der inhaltlichen Nähe des Testinstruments zum Paderborner Curriculum erklären und als Indikator für die Validität des Tests werten.

Kunina-Habenicht et al. (2013) analysierten im Rahmen des BilWiss-Projekts den Zusammenhang zwischen der Anzahl von besuchten bildungswissenschaftlichen Lehrveranstaltungen im Rahmen des Studiums und dem bildungswissenschaftlichen Professionswissen von Referendarinnen und Referendaren in Nordrhein-Westfalen. Das Professionswissen wurde bezogen auf verschiedene Inhaltsbereiche erfasst. Im Ergebnis zeigten sich entgegen der Erwartungen keine statistisch bedeutsamen Korrelationen zwischen der Anzahl besuchter Lehrveranstaltungen und den Inhaltsbereichen des bildungswissenschaftlichen Wissens. 
Fasst man die vorliegenden Forschungsbefunde zusammen, ist zu konstatieren, dass die angenommenen Zusammenhänge zwischen genutzten Lerngelegenheiten und fachlichen Wissensbeständen nicht in jeder Studie bestätigt werden konnten: In der TEDS-LT-Studie, der TEDS-M-Studie und in der Studie von Schmidt et al. (2011) werden positive Zusammenhänge zwischen den genutzten Lerngelegenheiten im Fach Mathematik und Facetten des mathematikspezifischen Professionswissen berichtet. Im Bereich der Bildungswissenschaften ist die Befundlage jedoch inkonsistenter. In der TEDS-LT-Studie erwies sich lediglich eine der Zeitvariablen als signifikanter Prädiktor für das pädagogische Wissen. Auch in der LEK-Studie wurden nur wenige der untersuchten Zusammenhänge zwischen wahrgenommenen Lerngelegenheiten und den erfassten Wissensfacetten signifikant. In der BilWiss Studie konnten keine signifikanten Zusammenhänge zwischen den belegten Veranstaltungen und dem bildungswissenschaftlichen Wissen nachgewiesen werden.

Als Erklärung für die uneinheitliche Befundlage kommen zum einen Unterschiede in der inhaltlichen Beziehung zwischen Testinstrument und Curriculum in Betracht. Für Mathematik konnten in mehreren Studien Zusammenhänge nachgewiesen werden, für die Bildungswissenschaften sind belegte Zusammenhänge eher die Ausnahme. Dies könnte daran liegen, dass mathematische Curricula über Hochschulstandorte hinweg vergleichbar sind als bildungswissenschaftliche Curricula und dass die Items in mathematischen Wissenstests eher hochschulübergreifend relevante mathematische Wissensfacetten abbilden, während sich bildungswissenschaftliche Testinstrumente eher auf lokale curriculare Schwerpunkte beziehen. Stehen die Lerngelegenheiten, wie z. B. die besuchten Lehrveranstaltungen und die belegten Module, dagegen in einem engeren inhaltlichen Zusammenhang mit dem erfassten Wissen, sind auch substanziellere Zusammenhänge für die Bildungswissenschaften anzunehmen, wie die Ergebnisse der LEK-Studie vermuten lassen.

Eine weitere Erklärung für die uneinheitliche Befundlage könnte in den unterschiedlichen Erfassungsmethoden von Lerngelegenheiten liegen. In der TEDS-LT-Studie wurde der zeitliche Studienaufwand als Indikator für genutzte Lerngelegenheiten herangezogen. Ein höherer Zeitaufwand muss aber nicht zwingend eine elaboriertere oder tiefgründigere Auseinandersetzung mit dem jeweiligen Lerngegenstand bedeuten, sondern kann im Gegenteil auch das Ergebnis von zusätzlich erforderlichen Wiederholungen, von Verständnisschwierigkeiten, ungünstigen Lernvoraussetzungen oder ineffizienten Lernstrategien sein. Auch aus der Hausaufgabenforschung sind solche negativen Zusammenhänge zwischen der individuell aufgewendeten Zeit - in dem Fall für Hausaufgaben - und den schulischen Leistungen bekannt (Schnyder, Niggli, Cathomas, Trautwein \& Lüdtke, 2006; Trautwein \& Lüdtke, 2007; Trautwein, Schnyder, Niggli, Neumann \& Lüdtke, 2009). Stancel-Piatak et al. (2013) selbst weisen bei der Interpretation ihrer inkonsistenten Befunde zur Bedeutung der Lernzeiten darauf hin, dass die Zunahme aufgewendeter Studienzeit auch die Folge ungüns- 
tiger Lernvoraussetzungen sein und durch Wiederholung nicht bestandener obligatorischer Lehrveranstaltungen zustande gekommen sein könnte, was die uneinheitlichen Zusammenhänge zwischen aufgewendeter Studienzeit und gemessenem Wissen zumindest teilweise erklären würde.

Als dritte Erklärung für die uneinheitliche Befundlage und nicht nachweisbare bzw. nicht substanzielle Zusammenhänge zwischen bildungswissenschaftlichen Lerngelegenheiten und pädagogischem bzw. bildungswissenschaftlichem Wissen kommt in Betracht, dass die genutzten Lerngelegenheiten nicht in ihrer Intensität und Qualität erfasst wurden. Dies führen auch die Autoren der BilWiss-Studie als möglichen Grund für die nicht signifikanten Zusammenhänge zwischen den wahrgenommenen Lerngelegenheiten in Form von besuchten Lehrveranstaltungen und den unterschiedlichen Facetten des bildungswissenschaftlichen Professionswissens an (Kunina-Habenicht et al., 2013).

Dieser Beitrag knüpft an diese genannten Problembereiche bei der Erfassung von Lerngelegenheiten an und führt die unterschiedlichen Herangehensweisen zur Erfassung von Lerngelegenheiten weiter. Im Rahmen der vorliegenden Studie wurde ein Instrument entwickelt, das zwischen der Behandlung von bildungswissenschaftlichen Themen und der Intensität der Auseinandersetzung differenziert. Mit dieser Vorgehensweise ist die Erwartung verbunden, dass das Zusammenspiel beider Informationen - das Vorhandensein einer Lerngelegenheit und die Intensität ihrer Nutzung - mehr zur Erklärung psychologisch-pädagogischen Wissens beitragen kann als die alleinige Berücksichtigung der angebotenen Lerngelegenheiten. In diesem Zusammenhang lässt sich in Anlehnung an die Angebots-Nutzungs-Modelle der Professionalisierungsforschung von Lehrpersonen (s.o.) annehmen, dass die vorkommenden Lerngelegenheiten nicht per se wirken, sondern über die Nutzung, hier die Intensität der Auseinandersetzung durch die Studierenden, vermittelt werden. Variiert wird in der Untersuchung zudem die thematische Passung zwischen Lerngelegenheiten und Items des Wissenstests, um die Veränderung in der Stärke des Zusammenhangs zu prüfen. Die Untersuchung wurde an einem Hochschulstandort, der Universität Kassel, durchgeführt.

\section{Fragestellungen und Hypothesen}

Die inkonsistente Forschungslage mit ihren zum Teil erwartungswidrigen Zusammenhängen aufgreifend, sollen in der vorliegenden Studie zwei Fragen untersucht werden: A) Wie ändert sich der Zusammenhang zwischen genutzten Lerngelegenheiten im bildungswissenschaftlichen Teil des Lehramtsstudiums und dem pädagogisch-psychologischem Wissen, wenn die inhaltliche Bindung zwischen den von den Studierenden genutzten Lerngelegenheiten und dem erfassten Wissen eher lose ist und wenn sie eher eng ist. Grundsätzlich wird ein schwacher positiver Zusammenhang zwischen der Anzahl der genutzten Lernge- 
legenheiten und dem pädagogisch-psychologischen Wissen der Studierenden erwartet, der enger werden sollte, wenn die Passung von Lerngelegenheiten und Testaufgaben stärker ausgeprägt ist. Demzufolge werden die folgenden beiden Hypothesen formuliert.

Hypothese (1): Die Anzahl aller genutzter Lerngelegenheiten im bildungswissenschaftlichen Studium sagt das pädagogisch-psychologische Wissen der Studierenden signifikant, aber in geringem Umfang, voraus.

Hypothese (2): Eine höhere Prädiktionskraft ergibt sich, wenn eine inhaltliche Passung der Lerngelegenheiten mit den erfragten Wissensbereichen des Leistungstests besteht.

B) Darüber hinaus wird danach gefragt, ob die Intensität der Beschäftigung mit Lerngelegenheiten eine Mediatorfunktion für den Einfluss der Lerngelegenheiten auf das Wissen hat.

Anknüpfend an die Angebots-Nutzungs-Modelle der Professionalisierungsforschung und bezugnehmend auf die o.g. These von Kunina-Habenicht et al. (2013), wonach auch der Intensität der Auseinandersetzung mit Lerngelegenheiten eine bedeutsame Rolle zur Erklärung des Wissens zukommen dürfte, wird folgende Hypothese formuliert:

Hypothese (3): Der Zusammenhang zwischen den Lerngelegenheiten und dem pädagogisch-psychologischen Wissen wird durch die Intensität der Auseinandersetzung mit diesen Lerngelegenheiten vermittelt.

\section{Methode}

Diese Studie ist eingebettet in das Projekt PROMETEUS (PRONET Meta-Evaluationsstudie), das wiederum ein Bestandteil des Kasseler Qualitätsoffensiveprojekts «Professionalisierung durch Vernetzung» (PRONET) ist. ${ }^{1}$ Das PROMETEUS-Projekt widmet sich innerhalb des PRONET-Gesamtvorhabens unterschiedlichen Evaluationsfragen und verknüpft hierbei unterschiedliche Datenquellen. Für diesen Beitrag werden Daten aus der sogenannten Studierendenbefragung herangezogen (Lipowsky \& Motyka, 2017). Sie zielt mit einer Kombination aus mehrkohortigem Längsschnitt- und Querschnittdesign darauf ab, Professionalisierungsprozesse im Rahmen des Studiums nachzeichnen und erklären zu können. Für die hier vorgestellte Studie wurden querschnittlich erfasste Daten herangezogen. Zum Einsatz kam in der Studierendenbefragung u.a. ein standortspezifisch entwickeltes Instrument zur Erfassung von Lerngelegenheiten im erziehungs- und gesellschaftswissenschaftlichen Kernstudium, dem bildungswissenschaftlichen Anteil des Kasseler Lehramtsstudiums.

Im Rahmen der Studierendenbefragung wurden im Sommersemester 2016 Studierende mittels Online-Fragebogen $\mathrm{zu}$ den behandelten Inhalten im bildungswissenschaftlichen Kernstudium befragt. Zudem wurde ein Test zum 
pädagogisch-psychologischen Professionswissen administriert. Die hier herangezogene Stichprobe setzt sich aus $N=109$ Studierenden zusammen, die bei der Erhebung im Sommersemester 2016 höchstens im sechsten Semester waren. ${ }^{2}$ Im Durchschnitt befanden sich die Befragten im 4. Fachsemester des Kernstudiums $(S D=1.50)$. Von den befragten Studierenden sind $73.4 \%$ weiblich. Sie absolvieren zu $15.6 \%$ das Lehramtsstudium für berufliche Schulen, zu 27.5 \% das Studium für das Lehramt an Haupt- und Realschulen und zu 56.9 \% das Studium für das Lehramt an Gymnasien. ${ }^{3}$

Im Folgenden werden die beiden zentralen Erhebungsinstrumente genauer erläutert.

Lerngelegenheiten. Zur Erfassung der Lerngelegenheiten wurde ein selbstentwickeltes Instrument eingesetzt. Bei der Entwicklung wurde die Modulprüfungsordnung des erziehungs- und gesellschaftswissenschaftlichen Kernstudiums der Universität Kassel herangezogen, um relevante Inhalte des bildungswissenschaftlichen Studiums zu extrahieren. Hierbei entstand eine Liste mit 36 Inhalten (z. B. Lerntheorien, Konfliktlösung im pädagogischen Feld oder Neue Medien), die jeweils mit Beispielen für Unterthemen indiziert wurden. $\mathrm{Zu}$ jeder Lerngelegenheit bzw. jedem Inhalt sollten die Befragten zwei Angaben machen: Sie sollten angeben, ob der jeweilige Inhalt im Kernstudium behandelt wurde (ja oder nein) und wie intensiv sie sich mit den Inhalten auseinandergesetzt haben. Hierzu wurden die Studierenden gebeten, die Intensität auf einer vierstufigen Likert-Skala von "gar nicht» bis «umfassend» anzugeben (siehe auch Abb. 1). Im einleitenden Fragetext wurde darauf hingewiesen, auch die ausseruniversitäre Beschäftigungsintensität mit einzubeziehen, um neben formalen auch nonformale und informelle Lerngelegenheiten zu berücksichtigen. Abbildung 1 zeigt beispielhaft einen Fragebogenausschnitt zur Lerngelegenheit Lernstrategien.

\begin{tabular}{|c|c|c|c|c|c|c|}
\hline & \multicolumn{4}{|c|}{$\begin{array}{l}\text { Wie intensiv haben Sie sich } \\
\text { mit diesem Thema beschäftigt? }\end{array}$} & \multicolumn{2}{|c|}{$\begin{array}{c}\text { Wurde dieses Thema } \\
\text { im Kernstudium } \\
\text { behandelt? }\end{array}$} \\
\hline & $\begin{array}{c}\text { gar } \\
\text { nicht }\end{array}$ & $\begin{array}{l}\text { ansatz- } \\
\text { weise }\end{array}$ & $\begin{array}{l}\text { einge- } \\
\text { hender }\end{array}$ & $\begin{array}{l}\text { um- } \\
\text { fassend }\end{array}$ & $\mathrm{Ja}$ & Nein \\
\hline $\begin{array}{l}\text { Lernstrategien } \\
\text { (Beispielsweise: Oberflächen- } \\
\text { vs. Tiefenstrategien, metako- } \\
\text { gnitive Strategien, ressourcen- } \\
\text { bezogene Strategien) }\end{array}$ & 0 & & & & & $\bigcirc$ \\
\hline
\end{tabular}

Abbildung 1: Ausschnitt des Fragebogens zur Erfassung von Lerngelegenheiten im Kernstudium 
Für die 109 Studierenden wurden zwei Summenscores der bisher im Kernstudium behandelten Themen gebildet. Zur Überprüfung der Hypothese 1 wurden alle 36 Lerngelegenheiten des bildungswissenschaftlichen Studiums herangezogen. Jede Ja-Antwort wurde mit 1 gewertet. Durchschnittlich berichten die Studierenden zum Zeitpunkt der Befragung über $M_{\text {OTL-Alle }}=21.57\left(S D_{\text {OTL-Alle }}=6.29\right)$ behandelte Inhalte im bildungswissenschaftlichen Studium (MinOTL-Alle $=10$; MaxOTL-Alle $=33$ ). Dies entspricht im Mittel rund $60 \%$ der erfassten Inhalte.

Zur Überprüfung der Hypothese 2 wurde nur eine Auswahl der 36 Lerngelegenheiten genutzt, nämlich jene, zu denen mindestens zwei inhaltlich korrespondierende Aufgaben im Wissenstest enthalten sind. Dies betrifft sieben der erfassten Lerngelegenheiten. Die Studierenden berichten, dass die Mehrzahl dieser sieben Inhalte behandelt wurde $\left(M_{\text {OTL-Auswahl }}=5.44\right.$, $\left.S D_{\text {OTL-Auswahl }}=1.47\right)$. Die Angaben streuen zwisch MinOTL-Auswahl $=1$ und MaxOTL-Auswahl $=7$.

Die Angaben zur Beschäftigungsintensität mit den einzelnen Lerngelegenheiten wurden so kodiert, dass die beiden Pole der Antwortoptionen "gar nicht" den Wert 0 und "umfassend» den Wert 3 erhielten (vgl. Abb. 1). Anschliessend wurden die Angaben für die 36 Inhalte und für die sieben Inhalte jeweils zu einem Mittelwert zusammengefasst. Für die 36 Inhalte ergab sich ein Intensitätsmittelwert von $M_{\text {OTL-Alle }}=1.42$ mit einer Standardabweichung von $S D_{\text {OTL-Alle }}=0.36$. Der Intensitätsmittelwert für die ausgewählten sieben Inhalte beträgt $M_{\text {OTL-Auswahl }}=1.75$, die Standardabweichung beträgt $S D_{\text {OTL-Auswahl }}=0.56$. Zur Überprüfung der Hypothesen wurden jeweils die beiden Summenscores für die behandelten Inhalte und die korrespondierenden Intensitätsmittelwerte zueinander in Beziehung gesetzt.

Pädagogisch-psychologisches Professionswissen. Zur Erfassung des pädagogisch-psychologischen Wissens kam der Pädagogische Wissenstest (PWT) zum Einsatz. Er wurde ursprünglich für das Kasseler STUVE-Projekt (Studienerfolg und Studienverlauf von Kasseler Lehramtsstudierenden) entwickelt und enthält 30 Fragen zu sechs Themenbereichen (siehe Tabelle 1). Es handelt sich hierbei weitgehend um Fragen, mit denen deklaratives Wissen, also "Wissen, dass» (Renkl, 2015), über wissenschaftliche Konzepte (z. B. Klassenführung, Bezugsnormorientierung, kooperatives Lernen, Big-FishLittle-Pond Effekt u.a.) erfasst wird (siehe Tabelle 2). Für die Fragen des PWT wurde ein geschlossenes Single Choice Antwortformat mit vier Distraktoren gewählt. Einige Items wurden hierbei in kontextualisierte Situationsbeschreibungen eingebettet. 
Tabelle 1: Themenbereiche und Beispielinhalte des PWT

\begin{tabular}{ll}
\hline Themenbereich & Beispielinhalt für eine Frage des Themenbereichs \\
\hline Leistung und Leistungsbewertung & Beurteilungsfehler \\
\hline Motivation und Lernen & Selbstbestimmungstheorie der Motivation \\
\hline Unterrichtsmethodik & Kooperatives Lernen \\
\hline Pädagogische Positionen & Humanismus \\
\hline Anforderungen des Beruffeldes & Umgang mit Heterogenität \\
\hline Lehrpersonenverhalten & Effektive Klassenführung \\
\hline
\end{tabular}

Der PWT wurde in Form eines Online-Tests mit Speed-Komponente administriert. Eine aktualisierte Kurzfassung des PWT mit 21 Aufgaben wurde im Sommersemester 2016 von der oben bereits genannten Stichprobe bearbeitet. Die Testitems wurden jeweils einzeln als Pflichtfragen dargeboten. Für die Beantwortung jedes Items wurde ein Zeitintervall vorgegeben, innerhalb dessen die Abgabe einer Antwort erfolgen sollte. Die Grenzen dieses Intervalls ergaben sich aus den Bearbeitungszeiten der Testitems im Vorgängerprojekt STUVE. Ein geringes Überschreiten der oberen Zeitgrenze wurde toleriert, ein Unterschreiten der unteren Grenze jedoch nicht, um die Wahrscheinlichkeit zu verringern, dass die Studierenden Antworten auswählen, ohne sich vorab Gedanken über die Fragen gemacht zu haben. Für die Auswertungen wurden nur jene Fälle herangezogen, bei denen der Wissenstest bis zur letzten Frage ausgefüllt wurde und eine plausible Beantwortungszeit von 10 bis 50 Minuten für die 21 Testitems resultierte. Die mittlere Bearbeitungszeit betrug $M=16.95$ Minuten $(S D=5.57)$ mit einem Range von Min $=10.08$ bis $\operatorname{Max}=48.67$ Minuten.

Für die Beurteilung der Modellgüte wurden Auswertungsempfehlungen nach Bond und Fox (2015) herangezogen. Die EAP/PV-Reliabilität beträgt für die Stichprobe .72, was als hinreichend gilt. Der klassische Item-Diskriminationswert beträgt durchschnittlich .39, der WMSQ der Items bewegt sich in einem akzeptablen Bereich von 0.84 bis 1.12 mit einem T-Wert von $T<=|2.5|$. Die Logits der Item-Schwierigkeit weisen einen Wert von 2.05 für das schwierigste Item und -1.82 für das leichteste Item auf. Die WLE-Reliabilität des Fähigkeitsschätzers liegt mit .71 in einem akzeptablen Bereich für die Punktschätzer der Personenfähigkeit. Die Varianz von $\sigma^{2}$ wle $=.90$ ist ebenfalls annehmbar. 
Tabelle 2: Beispielaufgaben des PTW und die korrespondierenden Lerngelegenheiten

\begin{tabular}{|c|c|c|c|}
\hline $\begin{array}{l}\text { Dimension } \\
\text { PWT }\end{array}$ & $\begin{array}{l}\text { Dimension } \\
\text { OTL }\end{array}$ & Testaufgabe & Lösung \\
\hline \multirow[t]{7}{*}{$\begin{array}{l}\text { Unterrichts- } \\
\text { methodik }\end{array}$} & $\begin{array}{l}\text { Unterrichts- } \\
\text { methoden }\end{array}$ & $\begin{array}{l}\text { Welche Aussage zum kooperativen Lernen im Schulun- } \\
\text { terricht ist zutreffend? }\end{array}$ & \\
\hline & & $\begin{array}{l}\text { Kooperatives Lernen in der Gruppe ist erfolgreich und erfüllt } \\
\text { seinen Zweck, wenn ... }\end{array}$ & \\
\hline & & $\begin{array}{l}\text {... stärkere Schüler/innen die Aufgabenbearbeitung gröss- } \\
\text { tenteils übernehmen, damit schwächere Schüler/innen am } \\
\text { Modell lernen können. }\end{array}$ & \\
\hline & & $\begin{array}{l}\text {... die Lehrkraft die Gruppe beobachtet, ohne sie zu } \\
\text { unterstützen oder zu moderieren. }\end{array}$ & \\
\hline & & $\begin{array}{l}\text {.. die Lehrkraft den Lernprozess der Gruppe steuert und } \\
\text { Schritt für Schritt anleitet. }\end{array}$ & \\
\hline & & $\begin{array}{l}\text {... die Interdependenz der Gruppe durch die Aufgaben- } \\
\text { stellung gewährleistet ist. }\end{array}$ & $\mathbf{X}$ \\
\hline & & $\begin{array}{l}\text {... die Gruppenmitglieder unabhängig voneinander einen } \\
\text { separaten Teil zur Erfüllung der Aufgabe bis zum Schluss } \\
\text { übernehmen können. }\end{array}$ & \\
\hline \multirow[t]{6}{*}{$\begin{array}{l}\text { Lehrper- } \\
\text { sonen- } \\
\text { verhalten }\end{array}$} & $\begin{array}{l}\text { Unterrichts- } \\
\text { qualität }\end{array}$ & $\begin{array}{l}\text { Welche der folgenden Verhaltensweisen lässt sich primär } \\
\text { bei Lehrpersonen mit einer effektiven Klassenführung } \\
\text { beobachten? }\end{array}$ & \\
\hline & & Solche Lehrpersonen haben Humor. & \\
\hline & & $\begin{array}{l}\text { Solche Lehrpersonen markieren klare Grenzen einzelner } \\
\text { Unterrichtsphasen. }\end{array}$ & $\mathbf{X}$ \\
\hline & & $\begin{array}{l}\text { Solche Lehrpersonen interessieren sich für die } \\
\text { Lösungswege der Schüler/innen. }\end{array}$ & \\
\hline & & Solche Lehrpersonen stellen anspruchsvolle Aufgaben. & \\
\hline & & $\begin{array}{l}\text { Solche Lehrpersonen greifen nach dem Motto } \\
\text { „Konfliktklärung hat Vorrang“ kleinere Konflikte und } \\
\text { Störungen auf und diskutieren sie mit den Schüler/innen. }\end{array}$ & \\
\hline
\end{tabular}

Um die Forschungsannahmen zu prüfen, wurden für die erste und zweite Hypothese Regressionsanalysen durchgeführt; die dritte Hypothese wurde mittels Mediationsanalyse untersucht. Aufgrund der geringen Fallzahl wurde von einer latenten Modellierung der Lerngelegenheiten abgesehen (Bühner, 2011). Für die Ermittlung der Signifikanz des Mediatoreffektes wurde ein Bootstrapping Verfahren gewählt. Diese Methode erweist sich im Gegensatz zu konventionellen Signifikanztests als robust gegenüber der Verletzung der Normalverteilungsannahme der Residuen des indirekten Effektes. Für das Schätzen des Konfidenzintervalls wurden mit Hilfe eines Bias-korrigierten Bootstrap-Verfahrens aus der Stichprobe 10.000 Teil-Stichproben gezogen (Geiser, 2010; Preacher \& Hayes, 2008). 


\section{Ergebnisse}

Mit der Hypothese 1 wurde angenommen, dass die Anzahl aller bisher behandelten Inhalte in den Bildungswissenschaften nur eine schwache Prädiktionskraft für das pädagogisch-psychologische Wissen der Kasseler Lehramtsstudierenden besitzt. Erwartungsgemäss zeigt sich nur ein schwacher positiver signifikanter Zusammenhang. Das Modell 1 (siehe Tabelle 3) kann mit $R^{2}=.04$ $(F(1,106)=5.9 ; p<.05, \beta=.23)$ nur einen geringen Anteil der Unterschiede im pädagogisch-psychologischen Wissen erklären.

\section{Tabelle 3: Regressionsanalyse zur Vorhersage der Testleistung durch alle} Lerngelegenheiten (Modell 1) und durch Lerngelegenheiten mit thematischer Passung (Modell 2)

\begin{tabular}{lccccc}
\hline & $B$ & $S E(B)$ & $\beta$ & $p$ & $R^{2}$ \\
\hline $\begin{array}{l}\text { Modell 1: } \\
\text { OTL-Alle }\end{array}$ & 0.034 & 0.014 & .230 & $<.05$ & \\
\hline $\begin{array}{l}\text { Modell 2: } \\
\text { OTL-Auswahl }\end{array}$ & 0.359 & 0.083 & .389 & $<.001$ & \\
\hline
\end{tabular}

Anmerkungen: $B=$ unstandardisierte Regressionsgewichte; $S E(B)=$ Standardfehler; $\beta=$ standardisierte Regressionsgewichte; ${ }^{* * *} p<.001,{ }^{* *} p<.01,{ }^{*} p<.05$; zweiseitig; $R^{2}=$ korrigierte Determinationskoeffizienten

Hypothese 2 besagt, dass das pädagogisch-psychologische Fachwissen stärker vorhergesagt wird, wenn zwischen Lerngelegenheiten und Wissenstest eine engere inhaltliche Verbindung besteht. Für die Prüfung dieser Hypothese gehen nur jene behandelte Inhalte in die Analyse ein, zu denen mindestens zwei inhaltlich korrespondierende Testaufgaben vorliegen (s.o.). Die entsprechende Regressionsanalyse zeigt, dass die Leistung im pädagogisch-psychologischen Wissenstest durch die Berücksichtigung thematisch passender Lerngelegenheiten ebenfalls positiv vorhergesagt wird $(\beta=.389, S E(B)=0.083)$. Das Modell 2 (siehe Tabelle 3) kann mit einem korrigierten $R^{2}=.14(F(1,106)=18.67, p<.05)$ deutlich mehr Varianz in der Testleistung aufklären als die Summe aller behandelten Lerngelegenheiten im Modell 1.

Mit Hypothese 3 wurde angenommen, dass der Zusammenhang zwischen behandelten universitären Inhalten und pädagogisch-psychologischem Wissen durch die Intensität der Auseinandersetzung vermittelt wird. Um diese Hypothese zu prüfen, wurde ein Mediatormodell getestet. Hierfür wurden die Werte für die reduzierte Anzahl an behandelten Inhalten und für die entsprechende Beschäftigungsintensität herangezogen. Wie Abbildung 2 zeigt, erweisen sich der Zusammenhang zwischen Lerngelegenheiten und darauf bezogener Beschäftigungsintensität $(\beta=.683, S E(B)=0.052 p<.001)$ wie auch der Zusammenhang zwischen Beschäftigungsintensität und der Leistung im Wissenstest 
$(\beta=.344, \operatorname{SE}(B)=0.114, p=.002)$ als hochsignifikant. Der vormals direkte Effekt zwischen den behandelten Lerngelegenheiten und der Testleistung (siehe Modell 2) wird durch den Einbezug des Mediators nicht mehr signifikant $(\beta=.154, \operatorname{SE}(B)=0.94, p=.101)$ und vollständig durch die Intensität der Auseinandersetzung vermittelt. Der indirekte Effekt wird mit einem $\beta=.235$ $(S E(B)=0.081)$ für ein $99 \%$ Konfidenzintervall $[0.099,0.371]$ hochsignifikant. Das Modell kann $46.6 \%$ der Varianz der Beschäftigungsintensität $\left(R^{2}=.466\right.$, $p<.001)$ sowie $21.4 \%$ der Unterschiede im pädagogisch-psychologischen Wissen der Studierenden aufklären $\left(R^{2}=.214, F(2,106)=14.18, p<.005\right)$.

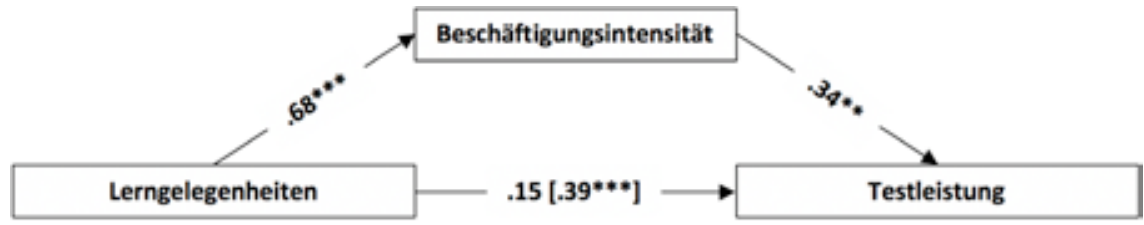

Abbildung 2: Mediator-Modell der besuchten universitären Lerngelegenheiten auf die Testleistung, mediiert durch die Beschäftigungsintensität

Anmerkungen: Indirekter Effekt basierend auf Bootstrapping: $\beta=.235, \operatorname{SE}(B)=0.081, p=.001$ (zweiseitig), KI = [0.099,0.371]; Koeffizienten sind standardisiert; Betagewicht in eckiger Klammer stellt den direkten Effekt ohne Mediator dar. $S E(B)=$ Standardfehler; KI = Konfidenzintervall [99 \%]; ${ }^{* * *} p<.001,{ }^{* *} p<.01,{ }^{*} p<.05$; zweiseitig

\section{Diskussion und Ausblick}

Die präsentierten Ergebnisse zeigen, dass der Zusammenhang zwischen Lerngelegenheiten und Wissen enger wird, sofern die Lerngelegenheiten eine thematische Passung mit dem erfragen Wissen aufweisen. So konnte gezeigt werden, dass die Berücksichtigung eines thematisch breiten Spektrums an Inhalten des bildungswissenschaftlichen Studiums der Universität Kassel nur einen schwachen Zusammenhang mit dem pädagogisch-psychologischen Wissen der Studierenden aufweist. Eine stärkere Vorhersagekraft haben die behandelten Inhalte, wenn ausschliesslich die im direkten inhaltlichen Zusammenhang mit den Fragen des Wissenstests stehenden Lerngelegenheiten herangezogen wurden. Für die Erklärung der uneinheitlichen Befundlage kann das Ergebnis insofern einen Beitrag leisten, als dass es verdeutlicht, dass die prognostische Validität von behandelten Lerngelegenheiten auch davon abhängig ist, wie eng die Lerngelegenheiten und das abhängige Kriterium inhaltlich zusammenhängen.

Dennoch ist dieses Ergebnis nicht sehr überraschend, denn es ist plausibel, dass Zusammenhänge zwischen zwei Variablen enger werden, wenn sie mehr inhaltliche Gemeinsamkeiten aufweisen. Gleichwohl ist zu beachten, dass der 
Titel einer Lehrveranstaltung oder die Behandlung eines Inhalts wenig über die Qualität der Auseinandersetzung und die Tiefe der kognitiven Verarbeitung aussagt und insofern nicht zwingend mit einem höheren Wissen einhergehen muss. So stellten König et al. (2012) in ihrer Studie fest, dass auch wenn der Titel einer Lehrveranstaltung eine enge Koppelung an das getestete Wissen vermuten lässt, nicht zwingend von einem signifikanten Zusammenhang zwischen den entsprechenden Lerngelegenheiten und dem erfassten Wissen ausgegangen werden kann.

Anknüpfend an die Angebots-Nutzungs-Modelle, die der Forschung für die Professionalisierung von Lehrpersonen vielfach zugrundeliegen, wurde in dieser Studie auch geprüft, ob sich die Intensität der Auseinandersetzung mit den Lerngelegenheiten tatsächlich als Mediator zwischen dem Angebot an Lerngelegenheiten und dem pädagogisch-psychologischen Wissen erweist. Tatsächlich wurde der vermittelnde Pfad signifikant. Der Zusammenhang zwischen den behandelten Studieninhalten und dem pädagogisch-psychologischen Wissen wird vollständig über die Intensität der Auseinandersetzung mediiert. Zudem zeigte sich, dass das Mediator-Modell mehr Varianz in der Testleistung erklärt als das Vorkommen von thematisch passenden Lerngelegenheiten allein. Demnach stellt nicht nur die Anzahl der behandelten Inhalte eine wichtige Determinante des Wissens der Studierenden dar, sondern auch die Intensität der Nutzung dieser Lerngelegenheiten. Damit lässt sich annehmen, dass die erfasste Intensität der Auseinandersetzung in Teilen offenbar auch die kognitive Verarbeitung der Studierenden widerspiegelt.

Insbesondere, wenn es - wie in der Hochschule - um selbstgesteuertes Lernen von Lernenden geht, sind mit Elliot (2015) komplexere Anforderungen an die Erfassung von Lerngelegenheiten zu stellen: Die Messung von OTL sollte nicht nur die Inhalte des intendierten Curriculums abbilden, sondern auch proximale Indikatoren für die kognitive Verarbeitung der entsprechenden Inhalte und die Intensität der Auseinandersetzung berücksichtigen. Nach den Ergebnissen dieser Studie sind tatsächlich weniger direkte Effekte der angebotenen Lerngelegenheiten und behandelten Lerninhalte zu erwarten als vielmehr indirekte Effekte, welche über die Tiefe der Nutzung vermittelt werden. Dies steht ganz im Einklang mit den Angebots-Nutzungs-Modellen der Lehrerinnen- und Lehrerforschung.

Wie jede Studie weist auch die vorliegende Untersuchung Grenzen auf. Grenzen dieser Studie stellen die Beschränktheit auf einen Hochschulstandort und die homogene Stichprobe dar. Damit sind die Ergebnisse zunächst nicht auf Kasseler Lehramtsstudierende der Grundschule und auf Studierende höheren Semesters übertragbar. Inwieweit die Befunde für andere Hochschulstandorte Gültigkeit beanspruchen können, muss ebenfalls zunächst offenbleiben. Zugleich bieten die Fokussierung auf einen Standort und der Einbezug einer homogenen Stichprobe aber auch bestimmte Vorteile. So konnten ein differenzierter Blick auf das spezifische Curriculum eingenommen und die Besonder- 
heiten des Kasseler Kernstudiums durch die Extraktion von einzelnen Inhalten berücksichtigt werden. Es wäre zu prüfen, inwiefern auch dieser differenzierte Zugang dazu beigetragen hat, den Mediationseffekt zwischen Lerngelegenheiten und Wissen aufzudecken und sichtbar zu machen.

Dass Lerngelegenheiten und Wissen gleichzeitig erfasst wurden, stellt eine weitere Grenze dieser Studie dar. Daher können die Befunde nicht als kausale Effekte interpretiert werden, auch wenn für den Wirkmechanismus - das Angebot an Lerngelegenheiten bestimmt die Nutzung, die sich wiederum auf das Wissen auswirkt - eine gewisse Plausibilität angenommen werden kann.

In diesem Zusammenhang stellt sich auch die Frage, welche Rolle motivationale Voraussetzungen der Studierenden, wie z. B. das Interesse und die Zielorientierungen, in diesem Wirkgefüge spielen. Erwartbar ist beispielsweise, dass das Interesse und die Lernzielorientierung den Zusammenhang zwischen Lerngelegenheiten und erworbenem Wissen weiter verstärken und moderierend wirken könnten. Auch kann unter Heranziehung weiterer Variablen analysiert werden, inwieweit die berichtete Nutzungsintensität der Studierenden mit weiteren individuellen Voraussetzungen der Studierenden, wie z. B. Geschlecht, Migrationshintergrund oder Abiturnote, kovariiert.

Unter Heranziehung weiterer Studierendenkohorten aus dem Kasseler PRONET-Projekt können weitere komplexere Fragestellungen geprüft werden, die mit der hier genutzten kleinen Stichprobe nicht beantwortet werden konnten. Hierzu gehört z. B. auch die Frage, wie sich das Professionswissen von bestimmten Subgruppen von Studierenden in Abhängigkeit von der Art der genutzten Lerngelegenheiten, deren Qualität und der Intensität ihrer Nutzung entwickelt. Mit einer grösseren Stichprobe lässt sich dann auch untersuchen, $\mathrm{ob}$ - bezogen auf die Behandlung und Nutzung der Studieninhalte - bestimmte Nutzungsprofile der Studierenden unterschieden werden können und inwiefern die Zugehörigkeit der Studierenden zu diesen Profilen die nachfolgende Entwicklung professionalisierungsrelevanter Variablen erklären kann. Annehmen lässt sich beispielsweise, dass Studierende, die sich vorwiegend und intensiv mit Inhalten bezogen auf das Thema Unterrichtsqualität auseinandergesetzt haben, über geringere Probleme im Schulpraktikum berichten und eher den Eindruck haben, dass sich Wissenschaft auf die Praxis beziehen lässt, als Studierende, die in ihrem Studium andere Schwerpunkt setzen.

Darüber hinaus ist in dem Evaluationsprojekt PROMETEUS geplant, in künftigen Erhebungen explizit zwischen den verschiedenen Kontexten formal, nonformal und informell zu unterscheiden, um deren Einflüsse getrennt voneinander untersuchen zu können. Das in dieser Studie eingesetzte Instrument zur Erfassung der Intensität der Auseinandersetzung mit bestimmten Inhalten unterscheidet nicht trennscharf zwischen diesen Kontexten. Zudem soll die von Studierenden eingeschätzte Qualität der angebotenen Lerngelegenheiten erfasst werden (vgl. auch Elliot, 2015). Die Metaanalyse von Schneider und Preckel (2017) gibt Hinweise darauf, welche Merkmale hierbei insbesondere in Betracht 
kommen. Einen der höchsten Effekte auf das Lernen der Studierenden $(d=1.35)$ erzielte die inhaltliche Klarheit (teacher's clarity and understandableness) und die kognitive Herausforderung bei der Vermittlung von bedeutungshaltigen Lerninhalten (stimulating meaningful learning) mit einem $d=0.65$ (ebd., S. 569 ff.). Insofern bieten sich die inhaltliche Klarheit und die kognitive Aktivierung als zwei wichtige Dimensionen an, um die qualitätsvolle und elaborierte Auseinandersetzung der Studierenden mit inhaltlichen Lerngelegenheiten genauer zu erfassen.

Welche Bedeutung hat diese Studie über den Standort Kassel hinaus? Neben den oben aufgeworfenen weiteren Forschungsfragen, die für die Forschung über den Standort Kassel hinaus relevant sind, zeigt die Studie, dass es lohnenswert ist, neben besuchten Lehrveranstaltungen und Modulen und behandelten Inhalten auch die Intensität der Auseinandersetzung durch die Befragung der Studierenden zu erfassen. Mit ähnlich gelagerten Studien an anderen Standorten könnte man prüfen, ob sich die hier berichteten Befunde replizieren lassen.

Von hochschulübergreifender Bedeutung ist zudem, dass selbstberichtete Lerngelegenheiten - in dieser Studie über behandelte Inhalte erfasst - auch in den Bildungswissenschaften keine irgendwie gearteten subjektiven Einschätzungen sind, sondern offenbar für erworbenes Wissen stehen und damit auch inhaltliche Validität beanspruchen können.

In diesem Zusammenhang ermöglicht die in dieser Studie realisierte Erfassung von Lerngelegenheiten auch die Bereitstellung von Daten für die Qualitätssicherung und -entwicklung im Rahmen eines Studiengangs (vgl. Darge, Schreiber, König \& Seifert, 2012). So können «blinde Flecken» im intendierten Curriculum identifiziert werden, also Themen, zu denen wenige Angebote bestehen und/oder die mit einer geringen Nutzungsintensität verbunden sind. In Kombination mit standardisierten Wissenstests eröffnet die Erfassung von Lerngelegenheiten ferner die Möglichkeit, die Effektivität von Studienelementen im Hinblick auf das implementierte Curriculum für den Aufbau von Professionswissen zu ermitteln (z. B. König et al., 2012). Dies ist insbesondere dann interessant, wenn Wahlmöglichkeiten bestehen und Studierende einzelne Module oder Veranstaltungen bewusst nicht in ihren Studienverlauf integrieren. Zudem erlaubt eine entsprechende Datengrundlage, Nutzungsprofile von Studierenden zu erstellen, um damit spätere Erfolgsmasse zu prädizieren (s.o.).

\section{Anmerkungen}

1 Das Projekt PRONET ist Teil des gemeinsamen Bund-Länder-Programms Qualitätsoffensive Lehrerbildung und wird aus Mitteln des Bundesministeriums für Bildung und Forschung gefördert.

2 Die Beschränkung auf Studierende, welche sich höchstens im sechsten Semester befinden, ist durch die mit den Wissenstestdaten durchgeführten Raschanalysen erklärbar. Hierbei wurde auch die Messinvarianz des pädagogisch-psychologischen Wissenstests für Studierende unterschiedlicher Semester mithilfe der Rasch-Tree-Methode nach Strobl, Kopf und Zeileis (2015) überprüft. Es konnte ermittelt werden, dass der Wissenstest für die Gruppe 
der Studierenden, welche sich im zweiten bis zum sechsten Studiensemester befanden, eine messinvariante Teststruktur aufwies. Für die Studierenden höherer Semester unterschied sich die ermittelte Teststruktur deutlich von der niedrigerer Semester.

3 Die Studierenden für das Lehramt an Grundschulen wurden wegen grösserer Unterschiede in der Prüfungsordnung und dadurch bedingter Unterschiede im intendierten Curriculum von dieser Analyse ausgeschlossen.

\section{Literatur}

Anderson, L. (1984). Time and school learning. Theory, research, and practice. New York, NY: St. Martin's Press.

Blömeke, S., Kaiser, G., Lehmann, R., König, J., Döhrmann, M., Buchholtz, C. \& Hacke, S. (2009). TEDS-M: Messung von Lehrerkompetenzen im internationalen Vergleich. In O. Zlatkin-Troitschanskaia, K. Beck, D. Sembill, R. Nickolaus \& R. Mulder (Hrsg.), Lehrerprofessionalität. Bedingungen, Genese, Wirkungen und ihre Messung (S. 181-209). Weinheim: Beltz Verlag.

Blömeke, S., Kaiser, G. \& Lehmann, R. (Hrsg.) (2010). TEDS-M 2008. Professionelle Kompetenz und Lerngelegenheiten angehender Mathematiklehrkräfte für die Sekundarstufe I im internationalen Vergleich. Münster: Waxmann.

Blömeke, S. \& König, J. (2010). Messung des pädagogischen Wissens: Theoretischer Rahmen und Teststruktur. In S. Blömeke, G. Kaiser \& R. Lehmann (Hrsg.), TEDS-M 2008. Professionelle Kompetenz und Lerngelegenheiten angehender Mathematiklehrkräfte für die Sekundarstufe I im internationalen Vergleich (S. 239-264). Münster: Waxmann.

Blömeke, S., Suhl, U., Kaiser, G. \& Döhrmann, M. (2012). Family background, entry selectivity and opportunities to learn: What matters in primary teacher education? An international comparison of fifteen countries. Teaching and Teacher Education, 28, (1), 44-55.

Bond, T. \& Fox, C. M. (2015). Applying the Rasch model: Fundamental measurement in the human sciences. New York, NY: Routledge.

Bühner, M. (2011). Einführung in die Test-und Fragebogenkonstruktion (3. Aufl.). München: Pearson.

Carroll, J. B. (1963). A model of school learning. Teachers College Record, 64, (8), 723-733.

Darge, K., Schreiber, M., König, J. \& Seifert, A. (2012). Lerngelegenheiten im erziehungswissenschaftlichen Studium. In J. König \& A. Seifert (Hrsg.), Lehramtsstudierende erwerben pädagogisches Professionswissen (S. 87-118). Münster: Waxmann.

Elliott, S. N. (2015). Measuring opportunity to learn and achievement growth: Key research issues with implications for the effective education of all students. Remedial and Special Education, 36, (1), 58-64.

Fend, H. (2002). Mikro- und Makrofaktoren eines Angebot-Nutzungsmodells von Schulleistungen. Zeitschrift für Pädagogische Psychologie, 16, (3-4), 141-149.

Geiser, G. (2010). Datenanalyse mit Mplus. Eine anwendungsorientierte Einführung. Wiesbaden: VS.

Harnischfeger, A. \& Wiley, D. E. (1977). Kernkonzepte des Schullernens. Zeitschrift für Entwicklungspsychologie und Pädagogische Psychologie, 9, (3), 207-228.

Hascher, T. \& Kittinger, C. (2014). Learning processes in student teaching. Analyses from a study using learning diaries. In K.-H. Arnold, A. Gröschner \& T. Hascher (Hrsg.), Schulpraktika in der Lehrerbildung. Theoretische Grundlagen, Konzeptionen, Prozesse und Effekte (S. 221-235). Münster: Waxmann.

Helmke, A. (2012). Unterrichtsqualität und Lehrerprofessionalität. Diagnose, Evaluation und Verbesserung des Unterrichts (4. Aufl.). Seelze: Kallmeyer.

Helmke, A. \& Renkl, A. (1992). Das Münchener Aufmerksamkeitsinventar (MAI): Ein Instrument zur systematischen Verhaltensbeobachtung der Schüleraufmerksamkeit im Unterricht. Diagnostica, 38, (2), 130-141. 
Husen, T. (1967). International study of achievement in mathematics: A comparison of twelve countries. New York, NY: Wiley.

König, J., Rothland, M. \& Schaper, N. (2018). Learning to practice, learning to reflect? Ergebnisse aus der Längsschnittstudie LtP zur Nutzung und Wirkung des Praxissemesters in der Lehrerbildung. Wiesbaden: Springer-Verlag.

König, J. \& Seifert, A. (2012). Der Erwerb von pädagogischem Professionswissen: Ziele, Design und zentrale Ergebnisse der LEK-Studie. In J. König \& A. Seifert (Hrsg.), Lehramtsstudierende erwerben pädagogisches Professionswissen (S. 7-31). Münster: Waxmann.

König, J., Tachtsoglou, S. \& Seifert, A. (2012). Individuelle Voraussetzungen, Lerngelegenheiten und der Erwerb von pädagogischem Professionswissen. In J. König \& A. Seifert (Hrsg.), Lehramtsstudierende erwerben pädagogisches Professionswissen (S. 234-283). Münster: Waxmann.

Kuger, S. (2016). Curriculum and learning time in international school achievement Studies. In S. Kuger, E. Klieme, N. Jude \& D. Kaplan (Hrsg.), Assessing contexts of learning: An international perspective (S. 395-422). Dordrecht: Springer.

Kunina-Habenicht, O., Schulze-Stocker, F., Kunter, M., Baumert, J., Leutner, D., Förster, D., Lohse-Bossenz, H. \& Terhart, E. (2013). Die Bedeutung der Lerngelegenheiten im Lehramtsstudium und deren individuelle Nutzung für den Aufbau des bildungswissenschaftlichen Wissens. Zeitschrift für Pädagogik, 59, (1), 1-23.

Kunter, M., Kleickmann, T., Klusmann, U. \& Richter, D. (2011). Die Entwicklung professioneller Kompetenz von Lehrkräften. In M. Kunter, J. Baumert, W. Blum, U. Klusmann, S. Krauss \& M. Neubrand (Hrsg.), Professionelle Kompetenz von Lehrkräften - Ergebnisse des Forschungsprogramms COACTIV(S. 55-68). Münster: Waxmann.

Kurz, A., Elliott, S. N., Lemons, C. J., Zigmond, N., Kloo, A. \& Kettler, R. J. (2014). Assessing opportunity-to-learn for students with disabilities in general and special education classes. Assessment for Effective Intervention, 40, (1), 24-39.

Lipowsky, F. (2014). Theoretische Perspektiven und empirische Befunde zur Wirksamkeit von Lehrerfort- und -weiterbildung. In E. Terhart, H. Bennewitz \&. M. Rothland (Hrsg.), Handbuch der Forschung zum Lehrerberuf(S. 511-541). Münster: Waxmann.

Lipowsky, F. \& Motyka, M. (2017). PRONET-Metaevaluationsstudie (PROMETEUS). In Zentrum für Lehrerbildung der Universität Kassel (Hrsg.), PRONET. Professionalisierung durch Vernetzung (S. 82-83). Universität Kassel. Verfügbar unter https://www.uni-kassel. de/themen/pronet/download.html

Mayr, J. (2012). Ein Lehramtsstudium beginnen? Ein Lehramtsstudium beginnen lassen? Laufbahnberatung und Bewerberauswahl konstruktiv gestalten. In B. Weyand, M. Justus \& M. Schratz (Hrsg.), Auf unsere Lehrerinnen und Lehrer kommt es an. Geeignete Lehrer/innen gewinnen, (aus-)bilden und fördern (S. 38-57). Essen: Stifterverband für die Deutsche Wissenschaft.

McDonnell, L. M. (1995). Opportunity to learn as a research concept and a policy instrument. Educational Evaluation and Policy Analysis, 17, (3), 305-322.

Preacher, K. J. \& Hayes, A. F. (2008). Asymptotic and resampling strategies for assessing and comparing indirect effects in multiple mediator models. Behavior Research Methods, 40, (3), 879-891.

Renkl, A. (2015). Wissenserwerb. In E. Wild \& J. Möller (Hrsg.), Pädagogische Psychologie (S. 3-24). Heidelberg: Springer.

Reusser, K. \& Pauli, C. (2010). Unterrichtsgestaltung und Unterrichtsqualität. Ergebnisse einer internationalen und schweizerischen Videostudie zum Mathematikunterricht Einleitung und Überblick. In K. Reusser, C. Pauli \& M. Waldis (Hrsg.), Unterrichtsgestaltung und Unterrichtsqualität. Ergebnisse einer internationalen und schweizerischen Videostudie zum Mathematikunterricht (S. 9-32). Münster: Waxmann.

Samuels, S. J. \& Turnure, J. E. (1974). Attention and reading achievement in first-grade boys and girls. Journal of Educational Psychology, 66, (1), 29-32. 
Schmidt, W. H., Cogan, L. \& Houang, R. (2011). The role of opportunity to learn in teacher preparation: An international context. Journal of Teacher Education, 62, (2), 138-153.

Schneider, M. \& Preckel, F. (2017). Variables associated with achievement in higher education: A systematic review of meta-analyses. Psychological Bulletin, 143, (6), 565-600.

Schnyder, I., Niggli, A., Cathomas, R., Trautwein, U. \& Lüdtke, O. (2006). Wer lange lernt, lernt noch lange nicht viel mehr: Korrelate der Hausaufgabenzeit im Fach Französisch und Effekte auf die Leistungsentwicklung. Psychologie in Erziehung und Unterricht, 53, (2), 107-121.

Stancel-Piatak, A., Abelha Faria, J., Dämmer, J., Jansing, B., Keßler, J.-U. \& Schwippert, K. (2013). Lerngelegenheiten und Veranstaltungsqualität im Studienverlauf: Lehramt Deutsch, Englisch und Mathematik. In S. Blömeke, A. Bremerich-Vos, G. Kaiser, G. Nold, H. Haudeck, J.-U. Keßler \& K. Schwippert (Hrsg.), Professionelle Kompetenzen im Studienverlauf. Weitere Ergebnisse zur Deutsch-, Englisch-und Mathematiklehrerausbildung aus TEDS-LT (S. 189-229). Münster und New York, NY: Waxmann.

Strobl, C., Kopf, J. \& Zeileis, A. (2015). Rasch trees: A new method for detecting differential item functioning in the Rasch model. Psychometrika, 80, (2), 289-316.

Trautwein, U. \& Lüdtke, O. (2007). Students' self-reported effort and time on homework in six school subjects: Between-students differences and within-student variation. Journal of Educational Psychology, 99, (2), 432-444.

Trautwein, U., Schnyder, I., Niggli, A., Neumann, M. \& Lüdtke, O. (2009). Chameleon effects in homework research: The homework-achievement association depends on the measures used and the level of analysis chosen. Contemporary Educational Psychology, 34, (1), 77-88.

Treiber, B. (1982). Lehr- und Lernzeiten im Unterricht. In B. Treiber \& F. Weinert (Hrsg.), Lehr-Lern-Forschung (S. 12-36). München: Urban \& Schwarzenberg.

Tynjälä, P. (2008). Perspective into learning at the workplace. Educational Research Review, 3, (2), 130-154.

\section{Schlagworte: Lerngelegenheiten, Lehrerprofessionalisierung, Fachwissen}




\section{Opportunités d'apprentissage et acquisition de connaissances en formation d'enseignants - une question d'intensité ?}

\section{Résumé}

Cet article examine l'impact des opportunités d'apprentissage sur les connaissances acquises en matière de psychologie de l'éducation en formation des enseignants. Notre étude conduite auprès d'un échantillon de 109 enseignants en formation à l'Université de Kassel montre que leurs connaissances en matière de psychologie de l'éducation peuvent être significativement prédites par les opportunités rencontrées dans les cours de travailler cette matière. La force de la prédiction est d'autant plus forte que les opportunités d'apprendre présentent des liens plus étroits en termes de contenu avec les connaissances à acquérir. Elle a permis de démontrer également que l'intensité avec laquelle les étudiants exploitent ces opportunités joue un rôle médiateur entre le nombre d'opportunités rencontrées et le niveau de maîtrise des connaissances atteint. La prise en considération simultanée des opportunités d'apprendre rencontrées dans le curriculum et de l'intensité du travail effectué par les étudiants explique en effet davantage de variabilité des acquis que la seule considération des opportunités rencontrées.

Mots-clés: Opportunités d'apprendre, professionnalisation des enseignants, connaissances acquises

\section{Opportunità d'apprendimento e acquisizione delle conoscenze nella formazione degli insegnanti: una questione d'intensità?}

\section{Riassunto}

Questo articolo esamina l'impatto delle opportunità d'apprendimento sulle conoscenze acquisite in psicologia dell'educazione nella formazione degli insegnanti. Il nostro studio, realizzato con un campione di 109 insegnanti in formazione all'Università di Kassel, mostra che le loro conoscenze in psicologia dell'educazione possono essere predette a partire dalle opportunità incontrate nei corsi per lavorare questa materia. La forza della prevedibilità aumenta quando le opportunità d'apprendimento portano su contenuti in stretto legame con le conoscenze da acquisire. Inoltre, lo studio ha permesso di dimostrare che l'intensità con la quale gli studenti sfruttano queste opportunità svolge un effetto di mediazione tra il numero di opportunità incontrate e il livello di padronanza delle conoscenze. Il fatto di considerare simultaneamente le opportunità d'apprendimento incontrate nei corsi di formazione e l'intensità del lavoro effet- 
tuato dagli studenti, permette di spiegare la maggiore variabilità delle conoscenze acquisite, che non la sola considerazione delle opportunità riscontrate.

Parole chiave: Opportunità d'apprendimento, professionalizzazione degli insegnanti, conoscenze acquisite

\section{The interrelation of opportunities to learn and professional knowledge in teacher training - a question of intensity?}

\section{Abstract}

This paper deals with the relationship between the learning opportunities in the educational science part of the teacher training program and the pedagogical and psychological professional knowledge of teacher training students. A study conducted on a sample of $\mathrm{N}=109$ student teachers from the University of Kassel shows that students' opportunities to learn in educational science captured as treated content - predict pedagogical-psychological knowledge, and that the predictive power increases when learning opportunities are more closely related to knowledge. In addition, based on the offer-and-use-model in professionalization research, it can be shown that the intensity of dealing with learning opportunities mediates the relationship between opportunities to learn and knowledge. The simultaneous consideration of opportunities to learn and intensity of a student's work on a given topic also explains more variance in pedagogical-psychological knowledge than the sole consideration of opportunities to learn.

Keywords: Opportunities to learn, teacher professional development, pedagogical-psychological knowledge, offer-and-use-model, mediator analysis 